\title{
ON THE RELATIVE SIZE OF TORIC BASES
}

\author{
CHRISTOS TATAKIS AND APOSTOLOS THOMA
}

\begin{abstract}
We consider the Graver basis, the universal Gröbner basis, a Markov basis and the set of the circuits of a toric ideal. Let $A, B$ be any two of these bases such that $A \nsupseteq B$ in general, we prove that there is no polynomial on the size or on the maximal degree of the elements of $A$ which bounds the size or the maximal degree of the elements of $B$ correspondingly.
\end{abstract}

\section{INTRODUCTION}

Let $A=\left\{\mathbf{a}_{1}, \ldots, \mathbf{a}_{m}\right\} \subseteq \mathbb{N}^{n}$ be a nonzero vector configuration in $\mathbb{Q}^{n}$ and $\mathbb{N} A:=\left\{l_{1} \mathbf{a}_{1}+\cdots+\right.$ $\left.l_{m} \mathbf{a}_{m} \mid l_{i} \in \mathbb{N}\right\}$ the corresponding affine semigroup. There are two cases for the semigroup $\mathbb{N} A$ either it is pointed, that is $\mathbb{N} A \cap(-\mathbb{N} A)=\{0\}$, or it is not pointed. We grade the polynomial ring $\mathbb{K}\left[x_{1}, \ldots, x_{m}\right]$ over an arbitrary field $\mathbb{K}$ by the semigroup $\mathbb{N} A$ setting $\operatorname{deg}_{A}\left(x_{i}\right)=\mathbf{a}_{i}$ for $i=$ $1, \ldots, m$. For $\mathbf{u}=\left(u_{1}, \ldots, u_{m}\right) \in \mathbb{N}^{m}$, we define the $A$-degree of the monomial $\mathbf{x}^{\mathbf{u}}:=x_{1}^{u_{1}} \cdots x_{m}^{u_{m}}$ to be

$$
\operatorname{deg}_{A}\left(\mathbf{x}^{\mathbf{u}}\right):=u_{1} \mathbf{a}_{1}+\cdots+u_{m} \mathbf{a}_{m} \in \mathbb{N} A,
$$

while we denote the usual degree $u_{1}+\cdots+u_{m}$ of $\mathbf{x}^{\mathbf{u}}$ by $\operatorname{deg}\left(\mathbf{x}^{\mathbf{u}}\right)$. The toric ideal $I_{A}$ associated to $A$ is the prime ideal generated by all the binomials $\mathbf{x}^{\mathbf{u}}-\mathbf{x}^{\mathbf{v}}$ such that $\operatorname{deg}_{A}\left(\mathbf{x}^{\mathbf{u}}\right)=\operatorname{deg}_{A}\left(\mathbf{x}^{\mathbf{v}}\right)$, see [15].

There are several sets for a toric ideal which include crucial information about it, such as the Graver basis, the Markov bases, the universal Gröbner basis and the set of the circuits. An irreducible binomial $\mathrm{x}^{\mathbf{u}}-\mathrm{x}^{\mathbf{v}}$ in $I_{A}$ is called primitive if there is no other binomial $\mathbf{x}^{\mathbf{w}}-\mathrm{x}^{\mathbf{z}}$ in $I_{A}$, such that $\mathbf{x}^{\mathbf{w}}$ divides $\mathbf{x}^{\mathbf{u}}$ and $\mathbf{x}^{\mathbf{z}}$ divides $\mathbf{x}^{\mathbf{v}}$. The set of the primitive binomials is finite, forms the Graver basis of $I_{A}$ and is denoted by $G r_{A}$. The universal Gröbner basis of an ideal $I_{A}$ is defined as the union of all reduced Gröbner bases $G_{<}$of $I_{A}$, as < runs over all term orders. It is a finite subset of the $I_{A}$ and it is a Gröbner basis for the ideal with respect to all admisible term orders, see [15]. The support of a monomial $\mathbf{x}^{\mathbf{u}}$ of $\mathbb{K}\left[x_{1}, \ldots, x_{m}\right]$ is $\operatorname{supp}\left(\mathbf{x}^{\mathbf{u}}\right):=\left\{i \mid x_{i}\right.$ divides $\left.\mathbf{x}^{\mathbf{u}}\right\}$ and the support of a binomial $B=\mathrm{x}^{\mathbf{u}}-\mathbf{x}^{\mathbf{v}}$ is $\operatorname{supp}(B):=\operatorname{supp}\left(\mathbf{x}^{\mathbf{u}}\right) \cup \operatorname{supp}\left(\mathbf{x}^{\mathbf{v}}\right)$. An irreducible nonzero binomial is called circuit if it has minimal support. The set of the circuits of a toric ideal $I_{A}$ is denoted by $\mathcal{C}_{A}$. A Markov basis is a minimal generating set of the toric ideal $I_{A}$, consisting of binomials, see [7, Theorem 3.1]. A relation between some of the above sets was given by B. Sturmfels in [15]:

Proposition 1.1. [15, Proposition 4.11] For any toric ideal $I_{A}$ it holds:

$$
\mathcal{C}_{A} \subseteq \mathcal{U}_{A} \subseteq G r_{A} .
$$

Every reduced Gröbner basis is a generating set of the toric ideal $I_{A}$ consisting of binomials, therefore it contains also a Markov basis. Thus the universal Gröbner basis and the Graver basis contain at least one Markov basis. The Graver basis contains all the Markov bases of $I_{A}$ if and only if the semigroup $\mathbb{N} A$ is pointed, see [5]. It is well known that the above inclusions may or may not be strict, see [15, Example 4.12]. In famous classes of ideals, the equality happens between some of the above bases and is combined with interesting geometric, combinatorial and homological properties. For example robust are those toric ideals for which the universal

2020 Mathematics Subject Classification. 14M25, 05C25, 05C38.

Key words and phrases. Toric ideals, toric varieties, Graver degrees, markov degrees, universal Groöbner degrees, circuit degrees. 
Gröbner basis is a Markov basis [2] and strongly robust are those toric ideals for which the Graver basis is a Markov basis, see [10, 17]. Lawrence toric ideals are strongly robust [15] but also toric ideals of non pyramidal self dual projective toric varieties are strongly robust [21]. For unimodular toric ideals all the elements in the Graver basis are circuits [1].

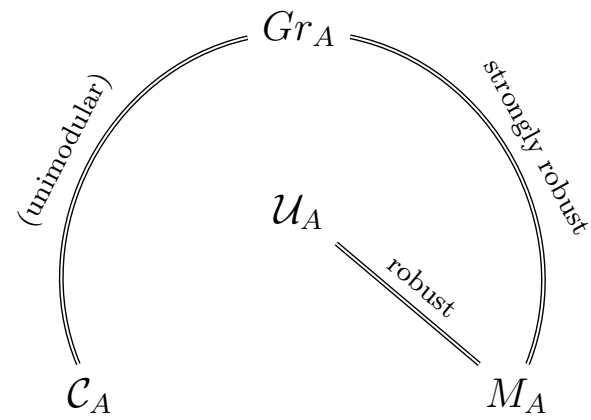

FiguRE 1. Well-known classes of toric ideals

There are several results in the literature concerning degree bounds of the elements of these sets and sometimes bounds on the one of these sets in terms of another set. There exist several bounds on the degrees of the elements of the Graver basis of a toric ideal which have important implications to integer programming and computational algebraic geometry, see for example [6, 8, 9, 13, 14, 15, 16, 19].

The aim of this article is to present several theorems concerning bounds on the size of these bases or the maximal degree of their elements in terms of the size or the maximal degree of the other bases. The proofs are based on carefully chosen simple counterexamples to show these relations with the fewest possible examples. Some of these examples are well known and we include them for completeness. There are also several other examples showing this extremal behavior.

In Section 2 we present the basic results about the toric ideals of graphs which will be useful for us in the sequel. For more details we refer to [12, 19, 22].

In Section 3 are the main results of the article that can be summarized in Figure 2. In Figure 2. $B \rightarrow C$ represents that the size of the base $B$ or the degrees of the elements of the set $B$ cannot be bounded above by a polynomial on the size or the maximal degree of the elements of $C$.

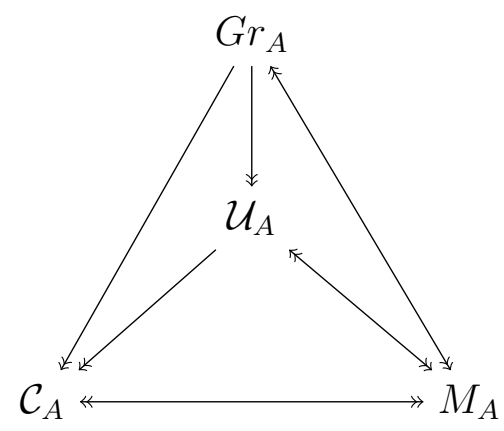

Figure 2. The relations of the size and the maximal degrees of the elements of the toric bases 


\section{ON THE TORIC BASES OF TORIC IDEALS OF GRAPHS}

Let $G$ be a finite simple connected graph with vertices $V(G)=\left\{v_{1}, \ldots, v_{n}\right\}$ and edges $E(G)=$ $\left\{e_{1}, \ldots, e_{m}\right\}$. Let $\mathbb{K}\left[e_{1}, \ldots, e_{m}\right]$ be the polynomial ring in the $m$ variables $e_{1}, \ldots, e_{m}$ over a field $\mathbb{K}$. We will associate each edge $e=\left\{v_{i}, v_{j}\right\} \in E(G)$ with the element $a_{e}=v_{i}+v_{j}$ in the free abelian group $\mathbb{Z}^{n}$ with basis the set of the vertices of $G$. Each vertex $v_{j} \in V(G)$ is associated with the vector $(0, \ldots, 0,1,0, \ldots, 0)$, where the nonzero component is in the $j$ position. We denote by $I_{G}$ the toric ideal $I_{A_{G}}$ in $\mathbb{K}\left[e_{1}, \ldots, e_{m}\right]$, where $A_{G}=\left\{a_{e} \mid e \in E(G)\right\} \subset \mathbb{Z}^{n}$.

A walk connecting $v_{i_{1}} \in V(G)$ and $v_{i_{s+1}} \in V(G)$ is a finite sequence of the form

$$
w=\left(\left\{v_{i_{1}}, v_{i_{2}}\right\},\left\{v_{i_{2}}, v_{i_{3}}\right\}, \ldots,\left\{v_{i_{s}}, v_{i_{s+1}}\right\}\right)
$$

with each $e_{i_{j}}=\left\{v_{i_{j}}, v_{i_{j+1}}\right\} \in E(G)$, for $j=1, \ldots, s$. A trail (respectively path) is a walk in which all edges (respectively vertices) are distinct. The length of the walk $w$ is the number $s$ of its edges. An even (respectively odd) walk is a walk of even (respectively odd) length. A walk $w=\left(\left\{v_{i_{1}}, v_{i_{2}}\right\},\left\{v_{i_{2}}, v_{i_{3}}\right\}, \ldots,\left\{v_{i_{s}}, v_{i_{s+1}}\right\}\right)$ is called closed if $v_{i_{s+1}}=v_{i_{1}}$. A cycle is a closed walk $\left(\left\{v_{i_{1}}, v_{i_{2}}\right\},\left\{v_{i_{2}}, v_{i_{3}}\right\}, \ldots,\left\{v_{i_{s}}, v_{i_{1}}\right\}\right)$ with $v_{i_{k}} \neq v_{i_{j}}$, for every $1 \leq k<j \leq s$.

Given an even closed walk $w$ of the graph $G$, where $w=\left(e_{i_{1}}, e_{i_{2}}, \ldots, e_{i_{2 q}}\right)$, we denote by $B_{w}$ the binomial

$$
B_{w}=E^{+}(w)-E^{-}(w)
$$

where $E^{+}(w)=\prod_{k=1}^{q} e_{i_{2 k-1}}, E^{-}(w)=\prod_{k=1}^{q} e_{i_{2 k}}$. It is known that the toric ideal $I_{G}$ is generated by binomials of this form, see [22]. Note that the binomials $B_{w}$ are homogeneous and the degree of $B_{w}$ is $q$, the half of the number of the edges of the walk. For convenience, we denote by $\mathbf{w}$ the subgraph of $G$ with vertices the vertices of the walk and edges the edges of the walk $w$. We call a walk $w^{\prime}=\left(e_{j_{1}}, \ldots, e_{j_{t}}\right)$ a subwalk of $w$ if $e_{j_{1}} \cdots e_{j_{t}} \mid e_{i_{1}} \cdots e_{i_{2 q}}$. An even closed walk $w$ is said to be primitive if there exists no even closed subwalk $\xi$ of $w$ of smaller length such that $E^{+}(\xi) \mid E^{+}(w)$ and $E^{-}(\xi) \mid E^{-}(w)$. The walk $w$ is primitive if and only if the binomial $B_{w}$ is primitive.

A cut edge (respectively cut vertex) is an edge (respectively vertex) of the graph whose removal increases the number of connected components of the remaining subgraph. A graph is called biconnected if it is connected and does not contain a cut vertex. A block is a maximal biconnected subgraph of a given graph $G$.

The following theorems determine the form of the circuits and the primitive binomials of a toric ideal of a graph $G$. R. Villarreal in [22, Proposition 4.2] gave a necessary and sufficient characterization of the circuits:

Theorem 2.1. Let $G$ be a graph and let $W$ be a connected subgraph of $G$. The subgraph $W$ is the graph $\mathbf{w}$ of a walk $w$ such that $B_{w}$ is a circuit if and only if

(1) $W$ is an even cycle or

(2) $W$ consists of two odd cycles intersecting in exactly one vertex or

(3) W consists of two vertex-disjoint odd cycles joined by a path.

The next theorem by E. Reyes et all, see [12], describes the form of the underlying graph of a primitive walk and thus describes the Graver basis of $I_{G}$.

Theorem 2.2. Let $G$ be a graph and let $W$ be a connected subgraph of $G$. The subgraph $W$ is the graph $\mathbf{w}$ of a primitive walk $w$ if and only if

(1) $W$ is an even cycle or

(2) $W$ is not biconnected and

(a) every block of $W$ is a cycle or a cut edge and

(b) every cut vertex of $W$ belongs to exactly two blocks and separates the graph in two parts, the total number of edges of the blocks that are cycles in each part is odd. 
We remark that every even primitive walk $w=\left(e_{i_{1}}, \ldots, e_{i_{2 k}}\right)$ partitions the set of the edges in the two sets $w^{+}=\left\{e_{i_{j}} \mid j\right.$ odd $\}$ and $w^{-}=\left\{e_{i_{j}} \mid j\right.$ even $\}$, otherwise the binomial $B_{w}$ is not irreducible. The edges of $w^{+}$are called odd edges of the walk $\mathrm{w}$ and those of $w^{-}$are called even. If $e_{i} \in w^{+}$and $e_{j} \in w^{-}$, we say that the edges $e_{i}$ and $e_{j}$ of the walk $w$ have different parity. Sink of a block $B$ is a common vertex of two odd or two even edges of the walk $w$ which belong to the block $B$. The last condition of Theorem 2.2 can be expressed also in terms of the walk as: every cut vertex of $\mathbf{w}$ belongs to exactly two blocks and it is a sink of both. A sink of a block should be always a cut vertex [12].

Afterwards, we recall from [12], some graph theoretical notions in order to describe a Markov basis of a toric ideal of a graph $G$. A binomial is called minimal, if it belongs to at least one minimal system of generators of $I_{G}$, i.e. at least one Markov basis of $I_{G}$.

For a given subgraph $F$ of $G$, an edge $f$ of the graph $G$ is called chord of the subgraph $F$, if the vertices of the edge $f$ belong to $V(F)$ and $f \notin E(F)$. A chord $e=\left\{v_{k}, v_{l}\right\}$ is called bridge of a primitive walk $w$ if there exist two different blocks $\mathcal{B}_{1}, \mathcal{B}_{2}$ of $\mathbf{w}$ such that $v_{k} \in \mathcal{B}_{1}$ and $v_{l} \in \mathcal{B}_{2}$. Let $w$ be an even closed walk $\left(\left\{v_{1}, v_{2}\right\},\left\{v_{2}, v_{3}\right\}, \ldots,\left\{v_{2 q}, v_{1}\right\}\right)$ and $f=\left\{v_{i}, v_{j}\right\}$ a chord of $w$. Then, $f$ breaks $w$ into two walks:

$$
w_{1}=\left(e_{1}, \ldots, e_{i-1}, f, e_{j}, \ldots, e_{2 q}\right) \text { and } w_{2}=\left(e_{i}, \ldots, e_{j-1}, f\right),
$$

where $e_{s}=\left\{v_{s}, v_{s+1}\right\}, 1 \leq s<2 q$ and $e_{2 q}=\left\{v_{2 q}, v_{1}\right\}$. The two walks are either both even or both odd. A chord is called even (respectively odd) if it is not a bridge and it breaks the walk into two even walks (respectively odd). A primitive walk $w$ is called strongly primitive if it has not two sinks with distance one in any cyclic block of $\mathbf{w}$.

The next theorem by Reyes et al, gives a necessary and sufficient characterization of the minimal binomials of a toric ideal of a graph $G$, thus describes the elements of Markov bases of $I_{G}$.

Theorem 2.3. [12, Theorem 4.13] Let $w$ be an even closed walk. $B_{w}$ is a minimal binomial if and only if

(M1) all the chords of $w$ are odd,

(M2) there are not two odd chords of $w$ which cross effectively except if they form an $F_{4}$,

(M3) no odd chord crosses an $F_{4}$ of the walk w,

(M4) $w$ is a strongly primitive.

The following theorem determines the indispensable elements of the ideal $I_{G}$, indispensable are the elements that belong to every Markov basis of $I_{G}$.

Theorem 2.4. [12, Theorem 4.14] Let $w$ be an even closed walk. $B_{w}$ is an indispensable binomial if and only if $w$ is a strongly primitive walk, all the chords of $w$ are odd and there are not two of them which cross effectively.

Finally, in order to describe the universal Gröbner basis for the case of toric ideals of graphs, we remind the notions of pure blocks and of the mixed walks of a graph $G$, see [19]. A cyclic block $\mathcal{B}$ of a primitive walk $w$ is called pure if all the edges of the block $\mathcal{B}$ belong either to $\mathbf{w}^{+}$ or to $\mathbf{w}^{-}$. A primitive walk $w$ is called mixed if none of the cyclic blocks of $w$ is pure. The next theorem describes completely the elements of the universal Gröbner basis of a toric ideal of a graph $G$.

Theorem 2.5. [19, Theorem 3.4] Let w be a primitive walk. $B_{w}$ belongs to the universal Gröbner basis of $I_{G}$ if and only if $w$ is mixed.

\section{SIZE AND DEGREE BOUNDS ON TORIC BASES}

3.1. On the comparison of the size of toric bases. Proposition 1.1 gives the comparison of the sets of $\mathcal{C}_{A}, \mathcal{U}_{A}, G r_{A}$ of a toric ideal. In the case of toric ideals of graphs, we know that 
every Markov basis of the ideal belongs also to its universal Gröbner basis, see [18, Proposition 3.3.], but this is not true in the general case, see [4, Example 1.8.]. In general, we have no information about the differences of the sizes between the above sets.

It is reasonable to ask about the comparison of the size of a Markov basis of the ideal with the subsets $\mathcal{C}_{A}, \mathcal{U}_{A}, G r_{A}$ of $I_{A}$. In this subsection, we answer the above question, studying the problem in two cases, i.e. the case that $\mathbb{N} A$ is pointed and the case that it is not.

3.1.1. Markov basis comparing to the sets of $\mathcal{C}_{A}, \mathcal{U}_{A}, G r_{A}$ of an ideal, where $\mathbb{N} A$ is pointed. Let $G$ be the graph of the Figure 3 with vertices $V(G)=\left\{v_{i}, u_{i} \mid 1 \leq i \leq\right.$ $n+1\} \cup\left\{s_{i}, t_{i}, x_{i}, y_{i} \mid 1 \leq i \leq n\right\}$ and edges

$$
\begin{aligned}
E(G)= & \left\{\left\{v_{i}, s_{i}\right\},\left\{s_{i}, t_{i}\right\},\left\{t_{i}, v_{i+1}\right\},\left\{v_{i}, v_{i+1}\right\},\left\{u_{i}, x_{i}\right\},\left\{x_{i}, y_{i}\right\},\left\{y_{i}, u_{i+1}\right\},\left\{u_{i}, u_{i+1}\right\} \mid 1 \leq i \leq n\right\} \\
& \cup\left\{\left\{v_{1}, u_{1}\right\},\left\{v_{n+1}, u_{n+1}\right\}\right\} .
\end{aligned}
$$

and let $I_{G}$ be its corresponding toric ideal.

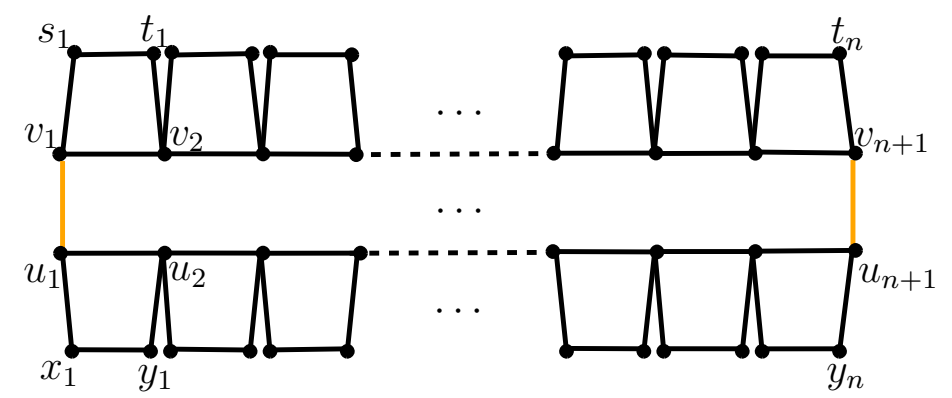

Figure 3. Example of a graph $G$ such that $\left|M_{G}\right| \ll\left|\mathcal{C}_{G}\right|=\left|\mathcal{U}_{G}\right|=\left|G r_{G}\right|$.

We use the above graph to prove the following theorem, see Figure 4

Theorem 3.1. The size of the elements of the Graver basis, the universal Gröbner basis and the set of the circuits of a toric ideal $I_{A}$ cannot be bounded above by a polynomial on the size of a Markov basis of $I_{A}$.

Proof. For the graph of the Figure 3 we claim that

$$
\left|M_{G}\right|=2 n+1 \text { and }\left|\mathcal{C}_{G}\right|=\left|\mathcal{U}_{G}\right|=\left|G r_{G}\right|=2 n+4^{n} \text {. }
$$

Note that the graph is bipartite with bipartition $(X, Y)$, where

and

$$
X=\left\{s_{i}, u_{i}, y_{i}, t_{j}, x_{j}, v_{j} \mid i \text { odd }, j \text { even }\right\}
$$

$$
Y=\left\{s_{j}, u_{j}, y_{j}, t_{i}, x_{i}, v_{i} \mid i \text { odd, } j \text { even }\right\} .
$$

It follows that the minimal generators of the corresponding toric ideal are exactly the binomials whose corresponding walks are the cycles of the graph $G$ with no chords. There are $2 n$ minimal generators of length four, in the forms $\left(v_{i}, s_{i}, t_{i}, v_{i+1}\right)$ or $\left(u_{i}, x_{i}, y_{i}, u_{i+1}\right)$ and one of length $2 n+2$, the cycle $\left(v_{1}, v_{2}, \cdots, v_{n+1}, u_{n+1}, u_{n}, \cdots, u_{2}, u_{1}\right)$. It follows that $\left|M_{G}\right|=2 n+1$.

Toric ideals of bipartite graphs are unimodular thus every element of the Graver basis of the ideal $I_{G}$ is also a circuit. By Proposition 1.1 it follows that $\left|\mathcal{C}_{G}\right|=\left|\mathcal{U}_{G}\right|=\left|G r_{G}\right|$. There are $2 n$ even cycles of length 4 and all other cycles are passing through both edges $\left\{v_{1}, u_{1}\right\},\left\{v_{n+1}, u_{n+1}\right\}$. From $v_{1}$ to $v_{n+1}$ there are $2^{n}$ different paths and from $u_{n+1}$ to $u_{1}$ there are also $2^{n}$ different paths. Therefore there are $2^{n} \cdot 2^{n}=4^{n}$ cycles passing through the edges $\left\{v_{1}, u_{1}\right\},\left\{v_{n+1}, u_{n+1}\right\}$. Thus $\left|\mathcal{C}_{G}\right|=\left|\mathcal{U}_{G}\right|=\left|G r_{G}\right|=2 n+4^{n}$.

In this case the semigroup $\mathbb{N} A_{G}$ is pointed, thus all Markov bases have the same size, actually in this example there exists only one Markov basis. Let $s=2 n+1$ be the size of the Markov basis 
then $\left|\mathcal{C}_{G}\right|=\left|\mathcal{U}_{G}\right|=\left|G r_{G}\right|=s-1+4^{(s-1) / 2}$. Therefore in this example the size of the elements of the Graver basis, the universal Gröbner basis and the set of the circuits is exponential on the size of a Markov basis of $I_{G}$. The result follows.

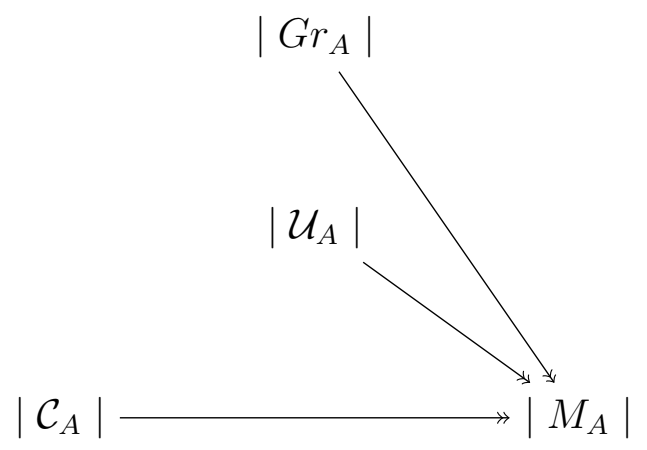

FiguRE 4. Size comparison between a Markov basis and all others

Remark 3.2. Note that for this example although the sizes of $\mathcal{C}_{G}, \mathcal{U}_{G}, G r_{G}$ cannot be bounded above by a polynomial on the size of a Markov basis of $I_{A}$, their maximal degrees have a linear relation, since $\operatorname{deg}_{M_{A}}=n+1$ and $\operatorname{deg}_{G r_{A}}=3 n+1$. Therefore, in this example the degrees of $\mathcal{C}_{G}, \mathcal{U}_{G}, G r_{G}$ can be bounded by a linear polynomial on $\operatorname{deg}_{M_{A}}$. Later on, we prove that this is not true in the general case, see Theorem 3.11 .

3.1.2. Markov basis comparing to the sets of $\mathcal{C}_{A}, \mathcal{U}_{A}, G r_{A}$ of an ideal, where $\mathbb{N} A$ is not pointed. We have the following theorem, see Figure 5:

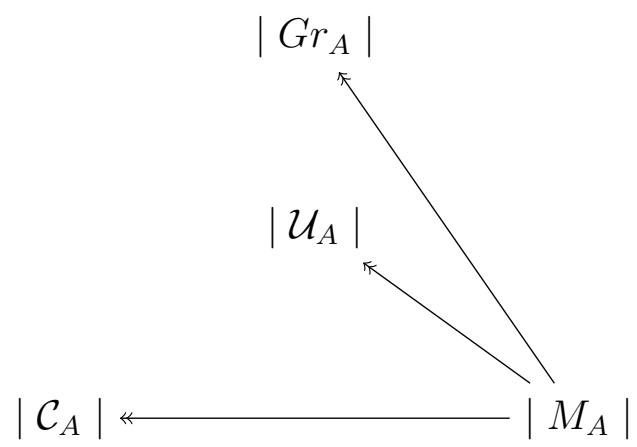

FiguRE 5. Size comparison between all others and a Markov basis

Theorem 3.3. The size of a Markov basis of $I_{A}$ cannot be bounded above by any function on the size of the Graver basis, the universal Gröbner basis and the set of the circuits of a toric ideal $I_{A}$.

Proof. Let $\mathbf{a}_{1}=1$ and $\mathbf{a}_{2}=-1$ then $\mathbb{N} A$ is the simplest example of a not pointed semigroup.

The toric ideal $I_{A}=\left\langle x_{1} x_{2}-1\right\rangle$ is principal and therefore $\mathcal{C}_{G}=\mathcal{U}_{G}=G r_{G}=\left\{x_{1} x_{2}-1\right\}$.

Let $a, b$ be positive integers and we consider $d=\operatorname{gcd}(a, b)$. Then both $x_{1}^{a} x_{2}^{a}-1$ and $x_{1}^{b} x_{2}^{b}-1$ are multiples of $x_{1}^{d} x_{2}^{d}-1$, since $d$ divides both $a, b$. Note also that $d$ can be expressed in the form $k a-l b$ or $l b-k a$ for some non negative integers. Then $x_{1}^{k a} x_{2}^{k a}-1-x_{1}^{d} x_{2}^{d}\left(x_{1}^{l b} x_{2}^{l b}-1\right)=x_{1}^{d} x_{2}^{d}-1$ or $x_{1}^{l b} x_{2}^{l b}-1-x_{1}^{d} x_{2}^{d}\left(x_{1}^{k a} x_{2}^{k a}-1\right)=x_{1}^{d} x_{2}^{d}-1$ and therefore

$$
\left\langle x_{1}^{a} x_{2}^{a}-1, x_{1}^{b} x_{2}^{b}-1\right\rangle=\left\langle x_{1}^{d} x_{2}^{d}-1\right\rangle .
$$


Using induction, the same formula is true for more than two integers.

Let $q_{1}, \ldots, q_{s}$ be pairwise relative prime integers greater than 1 . Let $Q=q_{1} \cdots q_{s}$ and $a_{i}=$ $Q / q_{i}$. Then $\left\langle x_{1}^{a_{1}} x_{2}^{a_{1}}-1, x_{1}^{a_{2}} x_{2}^{a_{2}}-1, \ldots, x_{1}^{a_{s}} x_{2}^{a_{s}}-1\right\rangle=\left\langle x_{1} x_{2}-1\right\rangle=I_{A}$ since the greatest common divisor of the $a_{1}, \ldots, a_{s}$ is one and

$$
\left.\left\langle x_{1}^{a_{j}} x_{2}^{a_{j}}-1\right| j \neq i \text { and } 1 \leq j \leq s\right\rangle=\left\langle x_{1}^{q_{i}} x_{2}^{q_{i}}-1\right\rangle \neq I_{A} .
$$

Therefore $\left\{x_{1}^{a_{1}} x_{2}^{a_{1}}-1, x_{1}^{a_{2}} x_{2}^{a_{2}}-1, \cdots, x_{1}^{a_{s}} x_{2}^{a_{s}}-1\right\}$ is a Markov basis. We have seen for the above example that the size of the Graver basis, the universal Gröbner basis and the set of the circuits of a toric ideal $I_{A}$ is one while there exist Markov bases of arbitrary large size.

This is a classical well known result. It shows easily an expected behavior of not pointed semigroups with respect to Markov bases. For more examples of not pointed semigroups and the properties of their Markov bases see [5].

Combining the results of Theorem 3.1 and Theorem 3.3 we have the Figure 6.

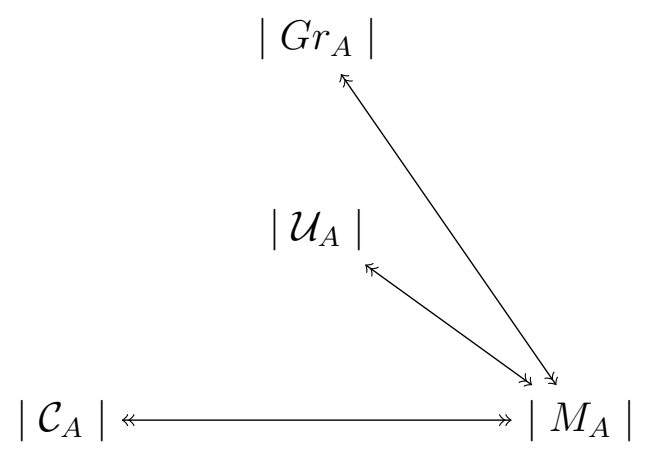

Figure 6. Comparison of the size of the toric bases

\subsection{On the degree bounds of toric bases.}

3.2.1. Maximal degree of the Graver basis comparing to the sets of $\mathcal{C}_{A}, \mathcal{U}_{A}, M_{A}$ of an ideal. Let $I_{A}$ be a toric ideal. For the rest of the paper, we denote by $\operatorname{deg}_{G r_{A}}, \operatorname{deg}_{\mathcal{U}_{A}}, \operatorname{deg}_{\mathcal{C}_{A}}, \operatorname{deg}_{M_{A}}$ the maximal degree of the elements of the Graver basis, the elements of the universal Gröbner basis, the circuits and the minimal generators of $I_{A}$, correspondingly.

Let $G_{1}, G_{2}$ be two vertex disjoint graphs, on the vertices sets $V\left(G_{1}\right)=\left\{v_{1}, \ldots, v_{s}\right\}, V\left(G_{2}\right)=$ $\left\{u_{1}, \ldots, u_{k}\right\}$ and on the edges sets $E\left(G_{1}\right), E\left(G_{2}\right)$ correspondingly. We define the sum of the graphs $G_{1}, G_{2}$ on the vertices $v_{i}, u_{j}$ as a new graph $G$ formed from their union by identifying the pair of vertices $v_{i}, u_{j}$ to form a single vertex $u$. The new vertex $u$ is a cut vertex in the new graph $G$ if both $G_{1}, G_{2}$ are not trivial. We say that we $a d d$ to a vertex $v$ of a graph $G_{1}$ a cycle $S$, to get a graph $G$ if $G$ is the sum of $G_{1}$ and $S$ on the vertices $v \in V\left(G_{1}\right)$ and any vertex $u \in S$ correspondingly.

Let $n$ be an odd integer greater than or equal to three. Let $G_{0}^{n}$ be a cycle of length $n$. For $r \geq 0$ we define the graph $G_{r}^{n}$ inductively on $r$. $G_{r+1}^{n}$ is the graph taken from $G_{r}^{n}$ by adding to each vertex of degree two of the graph $G_{r}^{n}$ a cycle of length $n$. Figure 7 shows the graph $G_{2}^{3}$.

We consider the graphs $G_{0}^{n}$ up to $G_{r-1}^{n}$ as subgraphs of $G_{r}^{n}$. We note that the graph $G_{r}^{n}$ is Eulerian since by construction it is connected and every vertex has even degree either four if it is also a vertex of $G_{r-1}^{n}$ or two if it is not. Let $w_{r}^{n}$ be any closed Eulerian trail of the graph $G_{r}^{n}$, i.e. a trail of the graph which visits every edge of the graph exactly once.

In [20] the authors proved that if $w_{r}^{n}$ is any closed Eulerian trail of the graph $G_{r}^{n}$, then the corresponding binomial $B_{w_{r}^{n}}$ is an element of the Graver basis of the ideal $I_{G_{r}^{n}}$ of degree

$$
\operatorname{deg}\left(B_{\left(w_{r}^{n}\right)}\right)=\frac{1}{2}\left(n+n^{2}\left(\frac{(n-1)^{r}-1}{n-2}\right)\right) .
$$




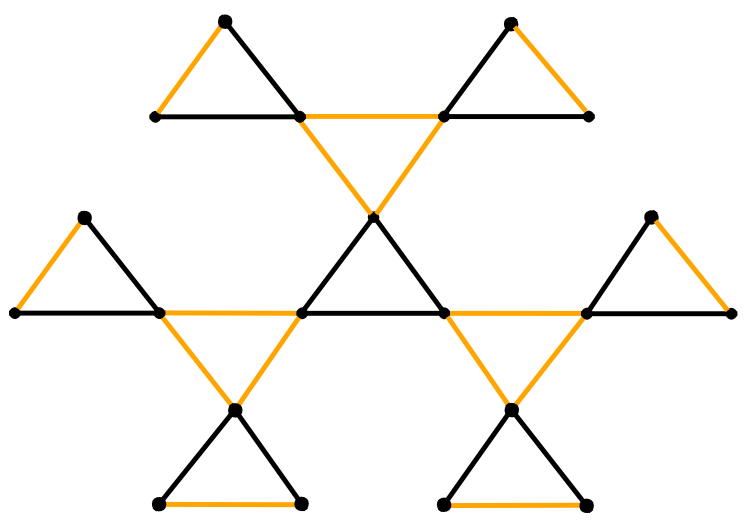

FiguRE 7. The Eulerian trail $w_{2}^{3}$ of the graph $G_{2}^{3}$

Moreover, they proved that the maximal degree of the circuits of the above graph is linear on $r$, i.e.

$$
\operatorname{deg}\left(C_{G_{r}^{n}}\right)=n+(2 r-1)(n-1),
$$

for more see [20, Proposition 4.3 and Remark 4.4].

We use these results to prove that the degree of an element in the Graver basis $G r_{A}$ of a toric ideal $I_{A}$ cannot be bounded above by a polynomial on the maximal degree of the elements of the universal Gröbner basis $\mathcal{U}_{A}$ and on the maximal degree of the elements of any Markov basis $M_{A}$ of the ideal too. To prove such a claim, we remark that for the graph $G_{r}^{3}$ the corresponding toric ideal is an example of an ideal in which the set of the circuits and the set of the elements of the universal Gröbner basis coincide, while the set of the minimal generators of the ideal is being included strictly on them as we can see in the next proposition. Note that the same result is not true if $n \neq 3$. To prove the next proposition we define the block tree of a graph $G$. Let $B(G)$ be the block tree of $G$, the bipartite graph with bipartition $(\mathbb{B}, \mathbb{S})$ where $\mathbb{B}$ is the set of blocks of $G$ and $\mathbb{S}$ is the set of cut vertices of $G,\{\mathcal{B}, v\}$ is an edge if and only if $v \in \mathcal{B}$.

Proposition 3.4. We consider the graph $G_{r}^{3}$ and let $I_{G_{r}^{3}}$ be its corresponding toric ideal. It holds

$$
M_{G_{r}^{3}} \subsetneq \mathcal{C}_{G_{r}^{3}}=\mathcal{U}_{G_{r}^{3}}
$$

Proof. Firstly, we will prove that $M_{G_{r}^{3}} \subsetneq \mathcal{C}_{G_{r}^{3}}$. It is enough to find an element of $\mathcal{C}_{G_{r}^{3}}$ which is not minimal. By definition, the graph $G_{r}^{3}$ includes the graph $G_{1}^{3}$ as a subgraph. By construction, the graph $G_{1}^{3}$ consists of four cycles and let it be $G_{1}^{3}=\left\{c_{1}, c_{2}=\left(e_{1}, e_{2}, e_{3}\right), c_{3}, c_{4}\right\}$. Clearly for the walk $w=\left(c_{1}, e_{1}, e_{2}, c_{3}\right)$ the corresponding binomial $B_{w}$ belongs to the $\mathcal{C}_{G_{r}^{3}}$. We remark that the edge $e_{3}$ is a chord of $w$ which is a bridge for the graph $\mathbf{w}$, which means that it is not an odd chord of $w$. It follows from Theorem 2.3 that $B_{w}$ is not minimal.

Next we will prove that $\mathcal{C}_{G_{r}^{3}}=\mathcal{U}_{G_{r}^{3}}$. By Proposition 1.1 we know that $\mathcal{C}_{G_{r}^{3}} \subseteq \mathcal{U}_{G_{r}^{3}}$. Let $B_{w}$ be an element of the $\mathcal{U}_{G_{r}^{3}}$, where $w$ is a primitive even closed walk of $G_{r}^{3}$. We will prove that $B_{w} \in \mathcal{C}_{G_{r}^{3}}$. From Theorem 2.5 it follows that the walk $w$ is mixed. Therefore every cyclic block of $\mathbf{w}$ is not pure. But every cycle is a 3-cycle which means that in every cyclic block of $\mathbf{w}$ two of the edges are in $\mathbf{w}^{+}$and one in $\mathbf{w}^{-}$or conversely. That means that each cyclic block has exactly one sink which means also exactly one cut vertex.

Let $n$ be the number of blocks of $\mathbf{w}, c$ the number of cut vertices and $s$ be the number of the cyclic blocks of $\mathbf{w}$, then $n-s$ is the number of cut edges. Every cut vertex belongs to exactly two blocks which means that $2 c$ is the number of edges in $B(\mathbf{w})$, the block tree of $\mathbf{w}$. The graph $B(\mathbf{w})$ is a tree therefore the number of vertices of $B(\mathbf{w})$ is the number of edges plus one. Thus $n+c=2 c+1$. It follows that there are $n-1$ cut vertices in $\mathbf{w}$. Every cut edge of $\mathbf{w}$ has two 
cut vertices and every cyclic block we have just proved has only one, therefore

$$
s+2(n-s)=2(n-1) .
$$

Therefore $s=2$ and that means $B_{w}$ is a circuit since $\mathbf{w}$ consists of two odd cycles and possible cut edges, see [22].

From the above proposition, we have the following theorem, see Figure 8 .

Theorem 3.5. The degrees of the elements in the Graver basis of a toric ideal $I_{A}$ cannot be bounded above by a polynomial on the maximal degree of the circuits, on the maximal degree of the elements of a Markov basis and on the maximal degree of the elements of the universal Gröbner basis of $I_{A}$.

Proof. We consider the graph $G_{r}^{3}$. From relation $\left(^{*}\right)$ it follows that $\operatorname{deg}\left(B_{w}\right)=9 \cdot 2^{r-1}-3$ for an element of maximal degree of the Graver basis of the ideal. Also, from relation $(* *)$ we have that $\operatorname{deg}_{\mathcal{C}_{G_{r}^{3}}}=4 r+1$. The result follows from Proposition 3.4 .

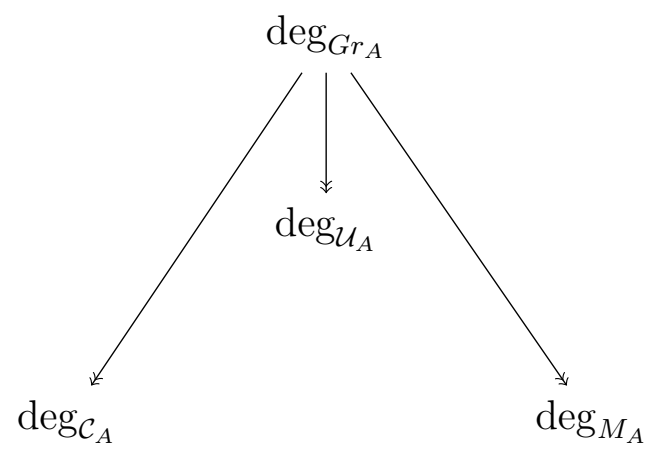

Figure 8. Comparison of degree bounds I

\subsubsection{Maximal degree of the circuits comparing to the sets of $G r_{A}, \mathcal{U}_{A}, M_{A}$ of an ideal.} Strongly robust toric ideals are ideals such that the Graver basis is a Markov basis. This implies that there is a unique Markov basis which is identical with any Gröbner basis, thus also with the universal Gröbner basis of $I_{G}$ as well as with the Graver basis. The only set that we do not have any information is the set of the circuits, except that it is a subset of the Graver basis. The next example shows that there may be huge difference between the size of these two sets.

We recall the definition of the subdivision of a graph. A $k$-subdivision of a graph $G$ is a new graph $S_{k}(G)$ taken from $G$ by replacing every edge of $G$ by $k$ new edges, where $k \geq 2$. Let $G=(V, E)$ then

$$
\begin{gathered}
V\left(S_{k}(G)\right)=V(G) \sqcup\left(\bigsqcup\left\{x(e)_{1}, \ldots, x(e)_{k-1} \mid e \in E\right\}\right) \\
E\left(S_{k}(G)\right)=\bigsqcup\left\{\left(u, x(e)_{1}\right),\left(x(e)_{1}, x(e)_{2}\right), \ldots,\left(x(e)_{k-1}, v\right) \mid e=(u, v) \in E\right\} .
\end{gathered}
$$

The new vertices $x(e)_{i}$ are all of degree two and this implies that any closed walk of $S_{k}(G)$ that passes through any one of $\left(u, x(e)_{1}\right),\left(x(e)_{1}, x(e)_{2}\right), \cdots,\left(x(e)_{k-1}, v\right)$ passes through all of them. Therefore there exists a one to one and onto correspondence between closed walks of $G$ and closed walks of $S_{k}(G)$. Let $w$ be a closed walk of $G$ of length $m$ then the corresponding closed walk $w_{k}$ of $S_{k}(G)$ has length $k m$ and has the property if $e=(u, v)$ is an edge of the walk $w$ then $\left(u, x(e)_{1}\right),\left(x(e)_{1}, x(e)_{2}\right), \cdots,\left(x(e)_{k-1}, v\right)$ are edges of $w_{k}$.

Proposition 3.6. Let $G$ be any graph and $S_{k}(G)$ the $k$ subdivision of $G$. The toric ideal $I_{S_{k}(G)}$ is strongly robust i.e.

$$
M_{S_{k}(G)}=\mathcal{U}_{S_{k}(G)}=G r_{S_{k}(G)}
$$


Proof. We will prove that $G r_{S_{k}(G)} \subseteq M_{S_{k}(G)}$. Let $B_{w_{k}} \in G r_{S_{k}(G)}$. Then $w_{k}$ is primitive and also strongly primitive since the distance of any two sinks in a cyclic block, if any, is a multiple of $k$. Also in $\mathbf{w}_{\mathbf{k}}$ there are no chords and certainly no $F_{4}$. It follows from Theorem 2.3 and Theorem 2.4 that $B_{w}$ is minimal and indispensable therefore there exists a unique Markov basis, thus $B_{w} \in M_{S_{k}(G)}$. We note that every Gröbner basis contains a Markov basis which implies that $G r_{S_{k}(G)} \subseteq M_{S_{k}(G)} \subseteq \mathcal{U}_{S_{k}(G)} \subseteq G r_{S_{k}(G)}$.

Remark 3.7. The graph $S_{k}(G)$ is an example of a graph which is robust, generalized robust (i.e. the union of all Markov bases of the ideal forms its universal Gröbner basis) and strongly robust, for more see [3, 17, 18]. We remark that the same result can be proved by using the bouquet algebra of toric ideals developed in [10]. Note that the edges $\left(u, x(e)_{1}\right),\left(x(e)_{1}, x(e)_{2}\right), \cdots,\left(x(e)_{k-1}, v\right)$ of $S_{k}(G)$ which correspond to the edge $(u, v)$ of $G$ belong to the same bouquet of the toric ideal $I_{S_{k}(G)}$, since every walk that passes through one of them passes through all of them and the corresponding bouquet is mixed since $k>1$. The result follows from [10, Corollary 4.4]. Note also that if $k$ is odd then a bouquet ideal of $I_{S_{k}(G)}$ is $I_{G}$.

The graph $S_{k}\left(G_{r}^{n}\right)$ is defined as a $k$-subdivision of the graph $G_{r}^{n}$, where $k$ is odd and $k \geq 3$. For example, we present the graph $S_{3}\left(G_{2}^{3}\right)$, see Figure 9 .

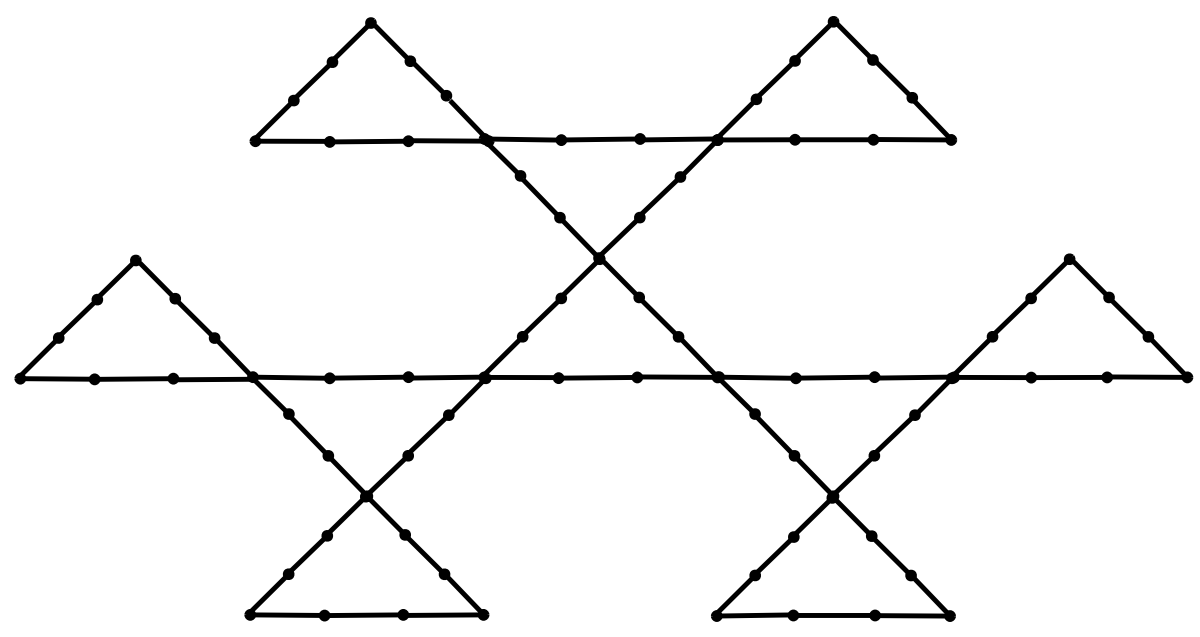

Figure 9. A 3-subdivision of the graph $G_{2}^{3}$

Proposition 3.8. We consider the graph $S_{k}\left(G_{r}^{n}\right)$ and let $I_{S_{k}\left(G_{r}^{n}\right)}$ be its corresponding toric ideal. For any closed Eulerian trail $\left(w_{k}\right)_{r}^{n}$ of the graph, we have that $B_{\left(w_{k}\right)_{r}^{n}} \in G r_{S_{k}\left(G_{r}^{n}\right)}$.

Proof. Let $\left(w_{k}\right)_{r}^{n}$ be a closed Eulerian trail of the graph $S_{k}\left(G_{r}^{n}\right)$, then $w_{r}^{n}$ is a closed Eulerian trail of the graph $G_{r}^{n}$. The graph of the walk $w_{r}^{n}$ is $G_{r}^{n}$ and the graph of the walk $\left(w_{k}\right)_{r}^{n}$ is $S_{k}\left(G_{r}^{n}\right)$. In [20, Proposition 4.1.], the authors proved for the graph $G_{r}^{n}$ that $B_{w_{r}^{n}}$ is an element of the Graver basis of $I_{G_{r}^{n}}$. The graph $S_{k}\left(G_{r}^{n}\right)$ is not biconnected and every block is a cycle. Also, if $v$ is a cut vertex of $S_{k}\left(G_{r}^{n}\right)$, it is also a cut vertex of $G_{r}^{n}$. The vertex $v$ separates the graph $G_{r}^{n}$ in two parts and let $m_{1}, m_{2}$ be the total number of the edges of the blocks that are cycles in each part correspondingly. Since the binomial $B_{w_{r}^{n}}$ is an element of the Graver basis, we remark that $m_{1}, m_{2}$ are odd. Obviously, the two parts of the graph $S_{k}\left(G_{r}^{n}\right)$, which separates the vertex $v$ have $k m_{1}, k m_{2}$ total number of edges correspondingly, i.e. an odd number. By Theorem 2.2 , it follows that the binomial $B_{\left(w_{k}\right)_{r}^{n}}$ belongs to the Graver basis of $I_{S_{k}\left(G_{r}^{n}\right)}$.

We consider the graph $B\left(S_{k}\left(G_{r}^{n}\right)\right)$ to be the block tree of $S_{k}\left(G_{r}^{n}\right)$. Let $\mathcal{B}_{k}, \mathcal{B}_{i}, \mathcal{B}_{l}$ be blocks of a graph $S_{k}\left(G_{r}^{n}\right)$. We call the block $\mathcal{B}_{i}$ an internal block of $\mathcal{B}_{k}, \mathcal{B}_{l}$, if $\mathcal{B}_{i}$ is an internal vertex in the unique path defined by $\mathcal{B}_{k}, \mathcal{B}_{l}$ in the block tree $B\left(S_{k}\left(G_{r}^{n}\right)\right)$. Every path of the graph 
$S_{k}\left(G_{r}^{n}\right)$ from the block $\mathcal{B}_{k}$ to the block $\mathcal{B}_{l}$ passes through every internal block of $\mathcal{B}_{k}, \mathcal{B}_{l}$. The path has vertices at least the cut vertices of $S_{k}\left(G_{r}^{n}\right)$ which are vertices in the path $\left(\mathcal{B}_{k}, \ldots, \mathcal{B}_{l}\right)$ in $B\left(S_{k}\left(G_{r}^{n}\right)\right)$.

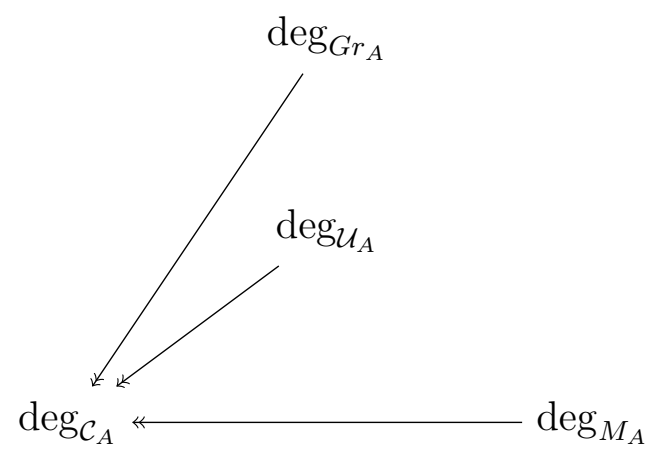

Figure 10. Comparison of degree bounds II

Theorem 3.9. The degrees of the elements of a Markov basis, the degrees of the elements of the universal Gröbner basis and the degrees of the elements of the Graver basis of a toric ideal $I_{A}$, cannot be bounded above by a polynomial on the degrees of the circuits of the ideal $I_{A}$.

Proof. We consider the graph $S_{k}\left(G_{r}^{n}\right)$ and let $I_{S_{k}\left(G_{r}^{n}\right)}$ be its corresponding toric ideal. Let $\left(w_{k}\right)_{r}^{n}$ be an Eulerian trail of the graph $S_{k}\left(G_{r}^{n}\right)$. By Proposition 3.8 we have that $B_{\left(w_{k}\right)_{r}^{n}}$ is an element of the Graver basis of $I_{S_{k}\left(G_{r}^{n}\right)}$. For the Eulerian trail $w_{r}^{n}$ of the graph $G_{r}^{n}$ from the relation $(*)$ we have that $\operatorname{deg}\left(B_{w_{r}^{n}}\right)=\frac{1}{2}\left(n+n^{2}\left(\frac{(n-1)^{r}-1}{n-2}\right)\right)$. Every edge of the walk $w_{r}^{n}$ corresponds to $k$ edges of the walk $\left(w_{k}\right)_{r}^{n}$ therefore

$$
\operatorname{deg}\left(B_{\left(w_{k}\right)_{r}^{n}}\right)=\frac{k}{2}\left(n+n^{2}\left(\frac{(n-1)^{r}-1}{n-2}\right)\right) .
$$

Which means that there exists an element in the Graver basis of $I_{S_{k}\left(G_{r}^{n}\right)}$ whose degree is exponential in $r$. By Proposition 3.6, it happens as well for the elements of the Markov basis and of the universal Gröbner basis of the ideal.

Let $B_{w}$ be a circuit of $I_{S_{k}\left(G_{r}^{n}\right)}$. The graph $S_{k}\left(G_{r}^{n}\right)$ has no even cycles and therefore the subgraph corresponding to a circuit consists of two different odd cycles joined by a path, see Theorem 2.1. We remark that every cycle of the graph $S_{k}\left(G_{r}^{n}\right)$ has length $k n$ and it is a block. We claim that a path between two blocks $\mathcal{B}_{1}, \mathcal{B}_{2}$ of $S_{k}\left(G_{r}^{n}\right)$ has length at most $(2 r-1) k(n-1)$. Each such path passes through all internal blocks of $\mathcal{B}_{1}, \mathcal{B}_{2}$ and no other and has at most $k(n-1)$ common edges with every one of them. We denote by $d\left(\mathcal{B}_{1}, \mathcal{B}_{2}\right)$ the number of the internal blocks of $\mathcal{B}_{1}, \mathcal{B}_{2}$. From [20, Lemma 4.2.] we know that $d\left(\mathcal{B}_{1}, \mathcal{B}_{2}\right) \leq 2 r-1$. Therefore the path has at most length $d\left(\mathcal{B}_{1}, \mathcal{B}_{2}\right) \cdot k(n-1) \leq(2 r-1) k(n-1)$. Thus the corresponding circuit has degree at most $k n+(2 r-1) k(n-1)$ which is linear on $r$.

Therefore the degree of an element in the Graver basis, of the Markov basis and of the universal Gröbner basis of the toric ideal $I_{A_{S_{k}\left(G_{r}^{n}\right)}}$ cannot be bounded above by a polynomial on the maximal degree of a circuit.

Remark 3.10. Note that one can use the main result of [20] and Lawrence liftings to prove the same result. Lawrence liftings provide a technique of constructing toric ideals which are strongly robust. For more on strongly robust ideals and a generalization of the Lawrence lifting technique one can read [10]. 
3.2.3. Maximal degree of the Markov basis comparing to the sets of $\mathcal{C}_{A}, \mathcal{U}_{A}, G r_{A}$ of an ideal. We recall the example of Subsection 3.1 .2 , where we are in the case that $\mathbb{N} A$ is not pointed. By looking at the maximal degree of the elements of the different bases we remark that the unique element in the Graver basis, the universal Gröbner basis and the set of the circuits of the toric ideal $I_{A}$ has degree 2 while the maximal degree of an element in a Markov basis can be arbitrary high. Therefore we have also the following theorem, see Figure 11;

Theorem 3.11. The degrees of the elements of a Markov basis of $I_{A}$ cannot be bounded above by any function on the maximal degree of the elements of the Graver basis, on the maximal degree of the elements of the universal Gröbner basis and on the maximal degree of the of the circuits of a toric ideal $I_{A}$.

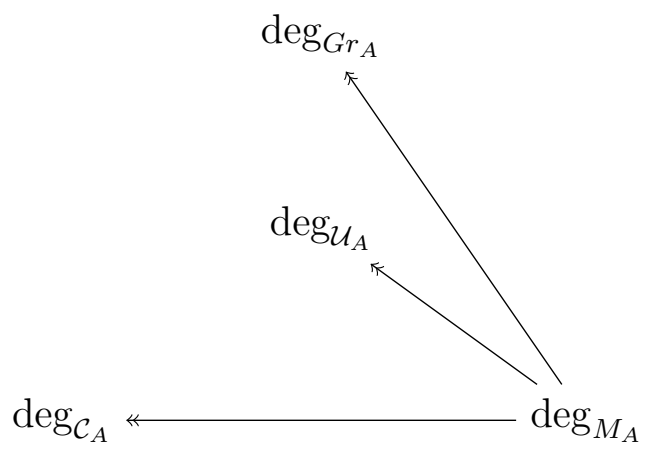

Figure 11. Comparison of degree bounds III

Returning in the case that $\mathbb{N} A$ is pointed, we consider the complete graph $G$. We note that a graph is complete if each pair of two distinct vertices of $G$, is connected by an edge. The complete graph with $n$ vertices is denoted by $K_{n}$. On this example we have the following theorem, see Figure 12:

Theorem 3.12. The degrees of the elements of the Graver basis, the degrees of the elements of the universal Gröbner basis and the degrees of the circuits of the ideal cannot be bounded above by any function on the maximal degrees of the elements of a Markov basis of the ideal.

Proof. In [19, Proposition 4.1], the authors proved that the largest degree $d_{n}$ of a binomial in the Graver basis (and in the universal Gröbner basis) for $I_{K_{n}}$ is $d_{n}=n-2$, for $n \geq 4$ and it is attained by a circuit, see also [6]. Furthermore, in [6], the authors proved that the maximal degree of the elements of a Markov basis of the ideal $I_{K_{n}}$ is two. The result follows.

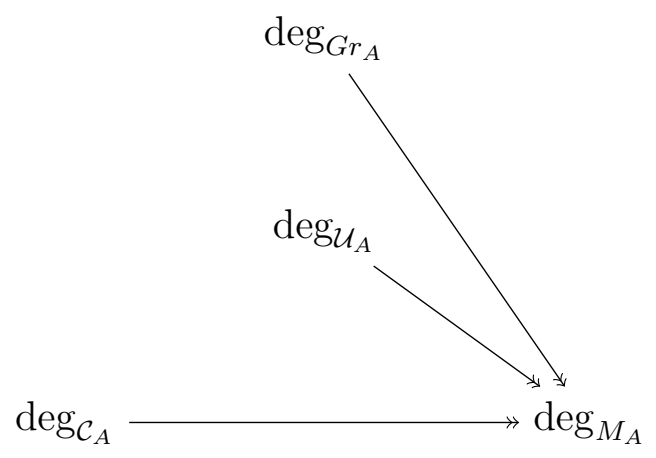

Figure 12. Comparison of degree bounds IV 
Combining the results of Theorem 3.5. Theorem 3.9. Theorem 3.11 and Theorem 3.12 we conclude them in Figure 13 .

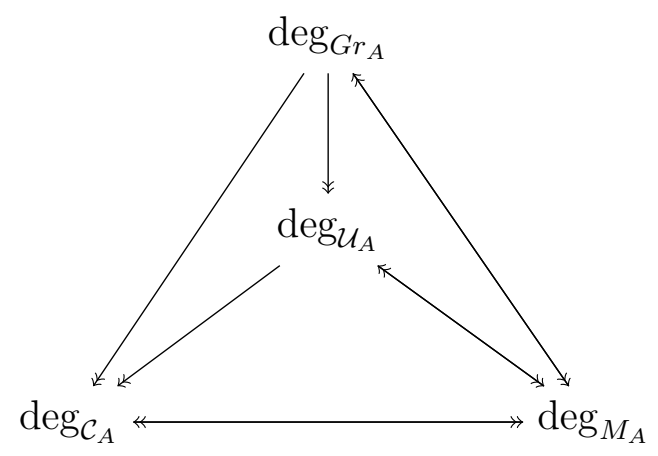

Figure 13. Comparison of degree bounds of toric bases

\section{Conclusion}

The main results of this manuscript are presented in Figure 6 and Figure 13, For the maximal degree of the elements of the sets: Graver basis, universal Gröbner basis, a Markov basis and the set of the circuits of a toric ideal we managed to prove that for any $A, B$ of these bases such that $A \nsupseteq B$ in general, there is no polynomial on the maximal degree of the elements of $A$ which bounds the maximal degree of the elements of $B$ correspondingly, for any possible combination. For the size we were able to provide the corresponding theorems in all cases except three, see Figure 6. Since maximal degree of a set can be considered as a measure of the size of this set we thought that the counterexamples used to prove the theorems in Subsection 3.2, may be also counterexamples in proving the missing theorems about the bounds of the size. But the form of the elements in these bases were so complicated that we were not be able to provide formulas for the actual size of these sets. Theorems [2.1]2.2, 2.3,2.5] suggest to use graphs like $G_{r}^{n}$ to produce counterexamples. The advantage of graphs like $G_{r}^{n}$ is that they increase the number of blocks exponentially on $r$ but make computation of all the elements even in the Graver basis very complicated. It seems to us that to find such counterexamples one has to move to a different class of toric ideals than the toric ideals of graphs.

Although most of the examples used in this article are toric ideals of graphs, one can use the theory of stable toric ideals and the generalized Lawrence matrices, developed in [10], to produce examples of more general toric ideals that they have exactly the same properties, since stable toric ideals preserve the size of toric bases and choosing all the vectors $c_{B}$ to have the same 1-norm $k$ all the degrees are multiplied with the constant $k$, for more details and examples see [10].

In [13] it was proved that for any toric ideal $I_{G}$ of a graph $G$ the degree of any element of the Graver basis of $I_{G}$ is bounded above by an exponential function of the maximal degree of a circuit. It is an interesting problem if this is true for any toric ideal. To prove something like that one needs a better understanding of circuits, Markov basis, universal Gröbner and Graver basis for general toric ideals or equivalently, in the case of pointed affine semigroups, for toric ideals of hypergraphs, see [11].

\section{REFERENCES}

[1] D. Bayer, S. Popescu, B. Sturmfels, Syzygies of unimodular Lawrence ideals, J. Reine Angew. Math. 534 (2001) 169-186.

[2] A. Boocher, E. Robeva, Robust toric ideals, J. Symbolic Computation 68:1 (2015) 254-264. 
[3] A. Boocher, B.Ch.Brown, T.Duff, L.Lyman, T.Murayama, A.Nesky, K.Schaefer, Robust graph ideals, Ann. Comb. 19 (2015) 641-660.

[4] H. Charalambous, A. Thoma, M. Vladoiu, Markov Bases and Generalized Lawrence Liftings, Ann. Comb. 19:4 (2015) 661-669.

[5] H. Charalambous, A. Thoma, M. Vladoiu, Minimal generating sets of Lattice Ideals, Collectanea Mathematica, 68 (2017) 377-400.

[6] J. De Loera, B. Sturmfels and R. Thomas, Gröbner bases and triangulations of the second hypersimplex, Combinatorica 15:3 (1995) 409-424.

[7] P. Diaconis, B. Sturmfels, Algebraic algorithms for sampling from conditional distributions, Ann. Statist. 26:1 (1998) 363-397.

[8] S. Hosten, Degrees of Gröbner bases of integer programs, Ph.D Thesis Cornell University (1997).

[9] S. Petrović, On the universal Gröbner bases of varieties of minimal degree, Math. Res. Lett. 15 (2008) 1211-1223.

[10] S. Petrović, A. Thoma, M. Vladoiu, Bouquet algebra of toric ideals, J. Algebra 512 (2018) 493-525.

[11] S. Petrović, A. Thoma, M. Vladoiu, Hypergraph encodings of arbitrary toric ideals, Journal of Combinatorial Theory, Series A 166 (2019) 11-41.

[12] E. Reyes, Ch. Tatakis, A. Thoma, Minimal generators of toric ideals of graphs, Adv. Appl. Math. 48 (2012) 64-78.

[13] K. Rychlewicz, A bound on degrees of primitive elements of toric ideals of graphs, Multigraded Algebra and Applications, Springer Proceedings in Mathematics and Statistics, NSA 2016, 125-131.

[14] Y.C. Stamatiou and Ch. Tatakis, An algorithm for computing the universal Gröbner basis of graph ideals, Int.J.Comp.Math.: Computer Systems Theory DOI: 10.1080/23799927.2019.1609585, 4:2 (2019) 67-75.

[15] B. Sturmfels, Gröbner Bases and Convex Polytopes. University Lecture Series, No. 8 American Mathematical Society.

[16] B. Sturmfels, Equations defining toric varieties, Algebraic Geometry, Santa Cruz 1995, American Mathematical Society, Providence, RI, (1997) 437-449.

[17] S. Sullivant, Strongly robust toric ideals in codimension 2, J. Algebraic Statistics 10:1 (2019) 128-136.

[18] Ch. Tatakis, Generalized robust toric ideals, J. Pure Appl. Algebra, 220 (2016) 263-277.

[19] Ch. Tatakis and A. Thoma, On the universal Gröbner bases of toric ideals of graphs, J. Combin. Theory Ser. A 118 (2011) 1540-1548.

[20] Ch. Tatakis and A. Thoma, Graver degrees are not polynomially bounded by true circuit degrees, J. Pure Appl. Algebra, 219 (2015) 2658-2665.

[21] A. Thoma and M. Vladoiu, The defining matrices of self dual projective toric varieties, preprint, 2021.

[22] R. Villarreal, Rees algebras of edge ideals, Comm. Algebra 23 (1995) 3513-3524.

Christos Tatakis, Department of Mathematics, University of Ioannina, Ioannina 45110, Greece Email address: chtataki@uoi.gr

Apostolos Thoma, Department of Mathematics, University of Ioannina, Ioannina 45110, GREECE

Email address: athoma@uoi.gr 